\title{
Myocardial Hypertrophy and the Maturation of Fatty Acid Oxidation in the Newborn Human Heart
}

\author{
MICHAEL A. YATSCOFF, JAGDIP S. JASWAL, MEGHAN R. GRANT, RACHEL GREENWOOD, TRISH LUKAT, \\ DONNA L. BEKER, IVAN M. REBEYKA, AND GARY D. LOPASCHUK
}

Departments of Pediatrics [M.A.Y., J.S.J., M.R.G., R.G., T.L., D.L.B., I.M.R., G.D.L.], and Surgery [D.L.B., I.M.R], University of Alberta, Edmonton, Alberta T6G 2S2, Canada

\begin{abstract}
After birth dramatic decreases in cardiac malonyl CoA levels result in the rapid maturation of fatty acid oxidation. We have previously demonstrated that the decrease in malonyl CoA is due to increased activity of malonyl CoA decarboxylase (MCD), and decreased activity of acetyl CoA carboxylase (ACC), enzymes which degrade and synthesize malonyl CoA, respectively. Decreased ACC activity corresponds to an increase in the activity of 5'-AMP activated protein kinase (AMPK), which phosphorylates and inhibits ACC. These alterations are delayed by myocardial hypertrophy. As rates of fatty acid oxidation can influence the ability of the heart to withstand an ischemic insult, we examined the expression of MCD, ACC, and AMPK in the newborn human heart. Ventricular biopsies were obtained from infants undergoing cardiac surgery. Immunoblot analysis showed a positive correlation between MCD expression and age. In contrast, a negative correlation in both ACC and AMPK expression and age was observed. All ventricular samples displayed some degree of hypertrophy, however, no differences in enzyme expression were found between moderate and severe hypertrophy. This indicates that increased expression of MCD, and the decreased expression of ACC and AMPK are important regulators of the maturation of fatty acid oxidation in the newborn human heart.

(Pediatr Res 64: 643-647, 2008)
\end{abstract}

$\mathrm{T}$ he adult heart has an extremely high energy demand which is met by the oxidation of fatty acids, accounting for $60-80 \%$ of cardiac ATP (ATP) production (1-5). Glycolysis, glucose oxidation, and lactate oxidation are responsible for the remainder of ATP production (2). Interestingly, cardiac energy substrate preference differs between the fetal and neonatal heart. In the fetal heart blood levels of fatty acids are low (6) and the heart has a low circulatory workload and meets its energy demands from glycolysis and lactate oxidation (6-8). At birth, major cardiovascular changes occur, including a reduction in pulmonary vascular resistance and closure of the ductus arteriosus and foramen ovale. These changes are coupled to an increase in peripheral vascular resistance, and an increased workload on the newborn heart. With circulating substrate and hormonal changes there is a switch in the energy substrate preference of the heart, from glucose and lactate metabolism to fatty acid oxidation (6-8). Although plasma fatty acid levels rapidly rise to levels seen in the

Received November 5, 2007; accepted June 24, 2008

Correspondence: Gary D. Lopaschuk, Ph.D., 423 Heritage Medical Research Centre, Departments of Pediatrics and Pharmacology, Faculty of Medicine and Dentistry, University of Alberta, Edmonton, Alberta, T6G 2S2; e-mail: gary.lopaschuk@ualberta.ca Supported by a grant from the Canadian Institutes of Health Research.

Supported by an Alberta Heritage Foundation for Medical Research Scientist (G.D.L.). adult immediately after birth (6), newborn rabbit studies have shown that the heart undergoes a transition from oxidizing glucose to oxidizing fatty acids during the $7 \mathrm{~d}$ immediately after birth $(9,10)$. The time frame responsible for the maturation of fatty acid oxidation in the human newborn heart is not yet known.

The increase in fatty acid oxidation after birth in the newborn rabbit heart is related to dramatic decreases in cardiac malonyl CoA levels $(10,11)$. Malonyl CoA is a potent inhibitor of carnitine palmitoyl transferase-1 (CPT-1), the rate limiting enzyme of mitochondrial fatty acid uptake (12). Acetyl CoA Carboxylase (ACC), is responsible for the synthesis of cardiac malonyl CoA (5,10,13-15) (Fig. 1), whereas malonyl CoA decarboxylase (MCD), is primarily responsible for the degradation of cardiac malonyl CoA (16). In newborn rabbit and pig hearts, a decrease in ACC activity and an increase in MCD activity is responsible for the decrease in malonyl $\mathrm{CoA}$ and the subsequent increase in fatty acid oxidation after birth $(10,16,17)$. The decrease in ACC activity is attributed to an increase in 5'-AMP activated protein kinase (AMPK) activity, which phosphorylates and inhibits ACC $(10,17,18)$ (Fig. 1). AMPK itself is inhibited by insulin (19), and in the newborn rabbit, a decrease in circulating levels of insulin after birth (6) disinhibits AMPK, thereby allowing it to phosphorylate and inhibit the activity of ACC (19), consequently decreasing the formation of malonyl CoA, and relieving the inhibition of mitochondrial fatty acid oxidation. Although circulating levels of insulin also decrease in newborn humans (6), it is not known what effect this has on the levels and phosphorylation state of cardiac ACC and AMPK. Thus, the roles of MCD, ACC, and AMPK in the maturation of fatty acid oxidation in the newborn human heart have yet to be determined.

Various pathophysiological states such as myocardial hypertrophy can influence cardiac fatty acid oxidation. In the adult, myocardial hypertrophy is associated with dramatic changes in energy metabolism, particularly a switch to a fetal glycolytic phenotype (20-22). In the newborn pig, myocardial hypertrophy delays the maturation of fatty acid oxidation due to persistently elevated malonyl CoA levels, elevated ACC activity, and decreased AMPK activity (17). This delay in the maturation of fatty acid oxidation may result in the newborn heart relying on glycolysis as a primary source of ATP, a metabolic shift also observed in the hypertrophied adult heart (20-22).

\footnotetext{
Abbreviations: AMPK, 5'-AMP activated protein kinase; ACC, acetyl CoA carboxylase; CPT 1, carnitine palmitoyl transferase 1; MCD, malonyl CoA decarboxylase; P-AMPK, phosphorylated 5'-AMP activated protein kinase
} 


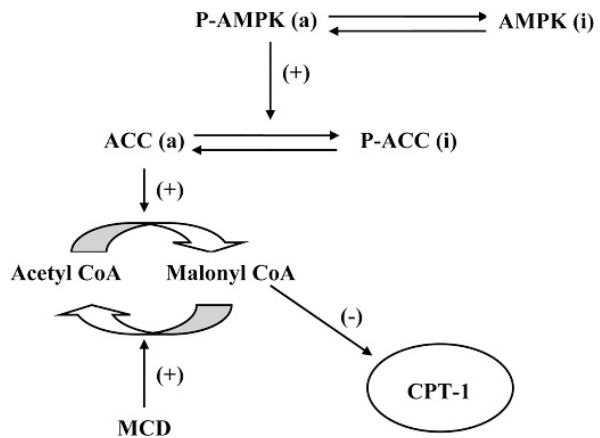

Figure 1. Regulation of fatty acid metabolism in the heart. ACC is inactivated upon phosphorylation by P-AMPK. Active ACC catalyzes the carboxylation of acetyl CoA to malonyl $\mathrm{CoA}$, which inhibits the rate-limiting enzyme in mitochondrial fatty acid uptake, CPT-1. MCD catalyzes the decarboxylation of malonyl CoA to acetyl CoA, removing the inhibition of CPT-1, (a) active form, $(i)$ inactive form, $(+)$ stimulates, $(-)$ inhibits.

Myocardial hypertrophy can occur in pediatric patients with congenital heart defects, such as tetralogy of fallot (TOF), hypoplastic left-heart syndrome (HLHS), atrioventricular septal defects (AVSD), ventricular septal defects (VSD), or right ventricular outflow tract obstructions (RVOTO). Whether myocardial hypertrophy alters the expression of the enzymes regulating malonyl CoA levels and, thus, the maturation of fatty acid oxidation in the newborn human heart is not known.

As energy substrate preference is an important determinant of the hearts ability to withstand an ischemic insult $(3,4,23-$ 25), and the newborn heart is subjected to a mandatory ischemic period during surgery to correct congenital heart defects, optimizing energy metabolism represents a novel therapeutic approach to protect the ischemic heart (23-25). However, before this potential therapeutic approach can be used in the newborn, it is important to have a better understanding of energy metabolism in the newborn human heart, particularly the maturation of fatty acid oxidation. The purpose of this study was to determine age-dependent changes in MCD, ACC, and AMPK expression in small ventricular biopsies collected at the time of cardiac surgery from 26 infants of varying age. Furthermore, this study examined the influence of varying degrees of cardiac hypertrophy on the enzymes regulating the maturation of fatty acid metabolism in the newborn human heart. In accordance with previous findings in animal models, we hypothesize that the maturation of fatty acid metabolism in the newborn human heart will be manifested as an increase in the expression of cardiac MCD, a decrease in the expression and/or increase in phosphorylation of ACC, as well as increased AMPK expression. We further hypothesize that cardiac hypertrophy will delay the increase in expression of MCD and AMPK, and thus delay the maturation of fatty acid oxidation in the newborn human heart.

\section{METHODS}

Sample collection. After legal guardian consent, 26 right ventricular myocardial samples ranging from 18 to $200 \mathrm{mg}$ in size were collected from infants undergoing cardiac surgery for the correction of congenital heart defects. The samples were obtained from specimens normally resected and discarded as part of the operative procedure. Surgeries were performed and samples collected at the University of Alberta Hospital, Walter C. Mackenzie Health Sciences Centre in Edmonton, Alberta, Canada. Ethical approval was obtained by the University of
Alberta Health Research Ethics Board, file \#4876. All tissues were immediately frozen in liquid $\mathrm{N}_{2}$ and stored at $-80^{\circ} \mathrm{C}$ until used for biochemical analysis.

Immunoblot analysis of MCD, ACC, and AMPK. For biochemical analysis, $25 \pm 2 \mathrm{mg}$ of tissue was homogenized in buffer containing $0.05 \mathrm{M}$ Tris- $\mathrm{HCl}$, $0.25 \mathrm{M}$ mannitol, $1 \mathrm{mM}$ EDTA, $1 \mathrm{mM}$ EGTA, $50 \mathrm{mM}$ sodium fluoride, $5 \mathrm{mM}$ sodium pyrophosphate, $1 \mathrm{mM}$ dithiotreitol, $0.1 \%$ (vol/vol) Protease Inhibitor for Mammalian Tissues (Sigma Chemical Co. Aldrich, Oakville, ON), 1\% (vol/vol) Phosphatase Inhibitor Cocktail I (Sigma Chemical Co.-Aldrich, Oakville, ON), Phosphatase Inhibitor Cocktail II (Sigma Chemical Co.-Aldrich, Oakville, ON), and $10 \%(\mathrm{wt} / \mathrm{vol})$ glycerol. Homogenates were cleared by centrifugation and the supernatant was collected for biochemical analysis. Protein was quantified using the Bradford method. Western immunoblots were performed as described previously (16). MCD was immunblotted with an antiMCD antibody (H2-40 antibody prepared in-house). ACC was immunblotted with a rabbit antiphospho-ACC (Ser79) antibody (Upstate Cell Signaling Technologies USA, Charlottesville, VA) or peroxidase-conjugated streptavivdin antibody (KPL, Gaithersburg, MA). AMPK was immunoblotted with antiPhospho-AMPK (Thr 172) antibody (Cell Signaling Technology, Inc., Beverly, MA), or antiAMPK antibody (Cell Signaling Technology, Inc., Beverly, MA).

Sample selection. As myocardial hypertrophy can decrease fatty acid oxidation in a manner proportional to its severity (26), samples were stratified based on the severity of hypertrophy. Hypertrophy was categorized as mild, moderate, and severe by the pediatric cardiac surgeon performing the corrective procedure by taking into account lesion type and patient age. When available, quantification of right ventricular wall thickness was performed via M-mode/two dimensional echocardiography, in either the long- or short-axis view, leading edge to leading edge at the equivalent level of the left ventricular papillary muscles at enddiastole. In instances where echocardiographic records were not available, hypertrophy was categorized solely according to lesion type and patient age.

Data analysis. Due to the large sample size, 3 gels were run for each enzyme of interest (MCD, P-ACC, ACC, P-AMPK, and AMPK). The possibility exists of slight variations in titers of the primary and secondary antibodies used in western immunoblotting. Consequently, the logarithm for all raw values was taken to account for any skewedness in the data (Figs. $2-4)$. Normalization for the mean \pm SD for each group was performed to eliminate slight differences between individual gels, thereby allowing direct comparison. This provided a sample size of 26 , with samples obtained from infants ranging in age from 0.2 to $10 \mathrm{mo}$. Age-dependent changes in enzyme expression were determined by linear regression analysis. Where appropriate, results are expressed as means \pm SEM. Differences in the expression of MCD, ACC, and AMPK between clinically moderate and severe hypertrophy were estimated by the Mann-Whitney $U$-test. Results were considered significant at $p<0.05$. All statistical analyses were performed using GraphPad InStat version 3.0 (GraphPad Software, San Diego, CA).

\section{RESULTS}

Patient demographics. A total of 26 right ventricular biopsies (10 samples from female patients and 16 samples from male patients) were obtained from newborn children $5 \mathrm{~d}(0.2 \mathrm{mo})$ to 10 mo of age undergoing surgical correction for either, VSD, HLHS, AVSD, TOF, or RVOTO (Table 1). These samples were used to assess age-dependent changes in the expression of MCD, as well

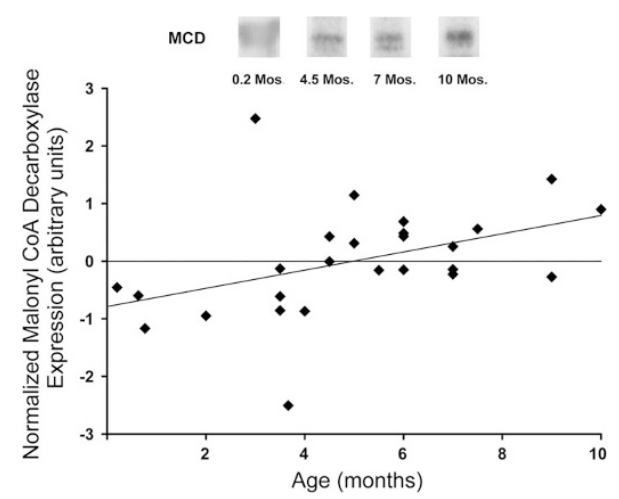

Figure 2. Normalized expression of MCD in right ventricular myocardial tissue from infants $0.2-10$ mo of age $(n=26)$ showing an age-dependant increase in the expression of $\mathrm{MCD}(r=0.42, p=0.03)$. Inset shows representative western immunoblots. 


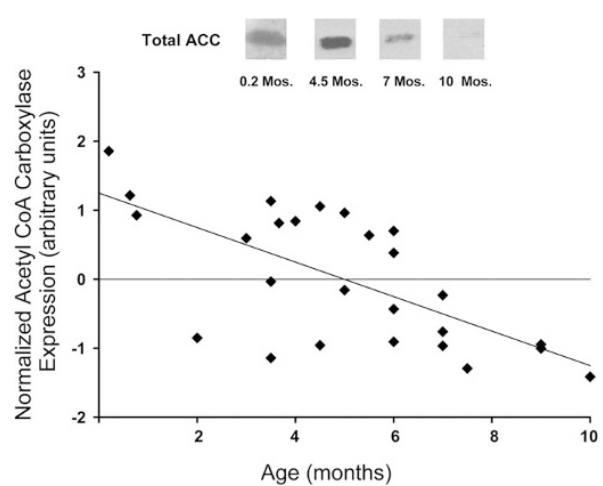

Figure 3. Normalized expression of ACC in right ventricular myocardial tissue from infants $0.2-10$ mo of age $(n=26)$ showing an age-dependant decrease in the expression of ACC $(r=-0.66, p=0.002)$. Inset shows representative western immunoblots.

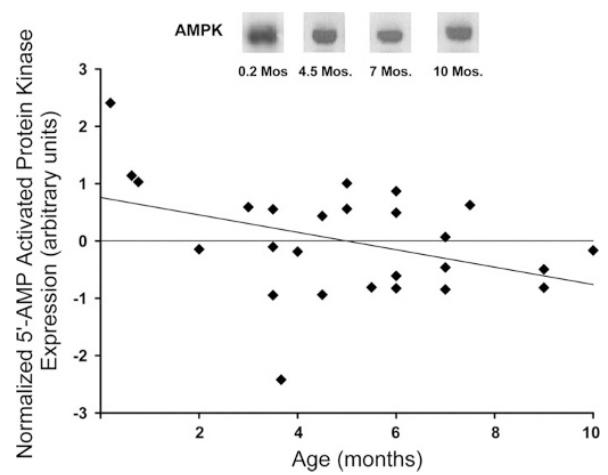

Figure 4. Normalized expression of AMPK in right ventricular myocardial tissue from infants $0.2-10$ mo of age $(n=26)$ showing an age-dependant decrease in the expression of AMPK $(r=-0.41, p=0.04)$. Inset shows representative western immunoblots.

as the age-dependent changes in the expression and phosphorylation of ACC and AMPK. All patients had some degree of myocardial hypertrophy. Patients with RVOTO were generally found to have severe hypertrophy (Table 1). A sub-group of patients $(n=5$ per group) of similar age (median age $=3.5 \mathrm{mo}$ ) with either moderate or severe hypertrophy were chosen to characterize the effects of myocardial hypertrophy on the expression of MCD, ACC, and AMPK (see below).

Age dependent changes in MCD expression. The effects of age on the expression of cardiac MCD, the enzyme responsible for the decarboxylation of malonyl CoA to acetyl CoA were first assessed. A significant $(p=0.03)$ age-dependant increase in expression of MCD was observed (Fig. 2), implying that as the infant matures, an increase in the expression of MCD likely decreases malonyl CoA content, and consequently disinhibits CPT-1, resulting in permissive effects on myocardial long-chain fatty acid oxidation. However, due to limited sample availability the levels of malonyl $\mathrm{CoA}$ in ventricular biopsies were not measured in this study.

Age-dependent changes in ACC expression and phosphorylation. The effects of age on the expression of cardiac ACC were next assessed. Although the heart expresses both a $265 \mathrm{kD}$ and $280 \mathrm{kD}$ isoform of ACC, the $280 \mathrm{kD}$ isoform predominates, and we were unable to detect the $265 \mathrm{kD}$ isoform by western immunoblotting. There was a significant $(p=0.002)$, agedependant decrease in the expression of ACC (Fig. 3), implying
Table 1. Demographic data of patients included for the study of the effects of age on the expression of $M C D$, and the expression and phosphorylation of $A C C$ and $A M P K$

\begin{tabular}{rlcll}
\hline $\begin{array}{c}\text { Age } \\
(\mathrm{mo})\end{array}$ & $\begin{array}{c}\text { Degree of } \\
\text { hypertrophy }\end{array}$ & $\begin{array}{c}\text { RV wall } \\
\text { thickness }(\mathrm{mm})\end{array}$ & Sex & $\begin{array}{c}\text { Clinical } \\
\text { condition }\end{array}$ \\
\hline 5.5 & Mild & 3.7 & $\mathrm{~F}$ & VSD \\
0.2 & Moderate & NA & $\mathrm{M}$ & HLHS \\
2.0 & Moderate & 4.1 & $\mathrm{M}$ & AVSD \\
3.0 & Moderate & 4.1 & $\mathrm{~F}$ & AVSD \\
3.5 & Moderate & 4.6 & $\mathrm{M}$ & VSD \\
4.5 & Moderate & 4.3 & $\mathrm{M}$ & TOF \\
6.0 & Moderate & 5.2 & $\mathrm{~F}$ & VSD \\
6.0 & Moderate & 4.6 & $\mathrm{~F}$ & HLHS \\
9.0 & Moderate & 4.8 & $\mathrm{M}$ & RVOTO \\
3.5 & Severe & 4.7 & $\mathrm{M}$ & RVOTO \\
3.5 & Severe & 4.1 & $\mathrm{M}$ & RVOTO \\
3.7 & Severe & NA & $\mathrm{F}$ & RVOTO \\
4.0 & Severe & 4.3 & $\mathrm{M}$ & RVOTO \\
4.5 & Severe & 5.9 & $\mathrm{M}$ & RVOTO \\
5.0 & Severe & 4.2 & $\mathrm{~F}$ & RVOTO \\
5.0 & Severe & NA & $\mathrm{F}$ & RVOTO \\
6.0 & Severe & 4.0 & $\mathrm{M}$ & TOF \\
6.0 & Severe & 5.2 & $\mathrm{M}$ & RVOTO \\
7.0 & Severe & NA & $\mathrm{M}$ & RVOTO \\
7.0 & Severe & 4.6 & $\mathrm{M}$ & RVOTO \\
7.0 & Severe & 4.9 & $\mathrm{M}$ & RVOTO \\
7.5 & Severe & 4.5 & $\mathrm{~F}$ & RVOTO \\
9.0 & Severe & 4.6 & $\mathrm{~F}$ & RVOTO \\
10.0 & Severe & NA & $\mathrm{M}$ & RVOTO \\
0.6 & Moderate-Severe & 4.9 & $\mathrm{M}$ & RVOTO \\
0.8 & Moderate-Severe & 4.8 & $\mathrm{~F}$ & RVOTO \\
\hline
\end{tabular}

Myocardial hypertrophy was assessed and classified according to patient age and lesion type as well as via echocardiographic analysis and categorized as ranging from moderate to severe.

F, female; M, male.

that as the heart matures, a decrease in ACC expression likely decreases the synthesis of malonyl CoA. As ACC can be phosphorylated at serine-79 and inhibited by AMPK, we also measured the levels of P-ACC using a phospho-specific antibody. There was no age-dependent change in the extent of ACC phosphorylation. This indicates that the synthesis of malonyl $\mathrm{CoA}$ and maturation of fatty acid oxidation is most likely regulated by the extent of ACC expression, and not the state of ACC phosphorylation in the newborn human heart.

Age-dependent changes in AMPK expression and phosphorylation. To further investigate this, the effects of age on the expression of AMPK were next assessed. There was a significant $(p=0.04)$ age-dependent decrease in the expression AMPK (Fig. 4). There was no age-dependent change in the extent of AMPK phosphorylation (activation) as determined by normalizing P-AMPK to the total amount of AMPK detected in each ventricular biopsy sample.

Effects of myocardial hypertrophy on the expression of $M C D$, and the expression and phosphorylation of ACC and AMPK. All ventricular biopsy samples in this study displayed varying degrees of cardiac hypertrophy (Table 1). The limited number of ventricular biopsy samples classified as clinically mild $(n=1)$ and moderate-severe hypertrophy $(n=2)$ precludes any statistical analysis of possible changes in the expression of MCD, ACC and AMPK. As such the effects of hypertrophy on MCD, ACC and AMPK expression were limited to ventricular biopsy 
Table 2. Demographic data of patients with moderate and severe myocardial hypertrophy selected for the assessment of the effects of hypertrophy on the expression of $M C D$, and the expression and phosphorylation of $A C C$ and $A M P K$

\begin{tabular}{lclc}
\hline & Age (mo) & Sex & Clinical condition \\
\hline Group A: moderate hypertrophy* & 2.0 & $\mathrm{~F}$ & AVSD \\
& 3.0 & $\mathrm{~F}$ & AVSD \\
& 3.5 & $\mathrm{M}$ & VSD \\
& 4.5 & $\mathrm{M}$ & TOF \\
Group B: severe hypertrophy $\dagger$ & 6.0 & $\mathrm{~F}$ & HLHS \\
& 2.0 & $\mathrm{~F}$ & RVOTO \\
& 3.5 & $\mathrm{M}$ & RVOTO \\
& 3.5 & $\mathrm{M}$ & RVOTO \\
& 4.0 & $\mathrm{M}$ & RVOTO \\
& 5.0 & $\mathrm{~F}$ & RVOTO
\end{tabular}

Myocardial hypertrophy was assessed as described under the section "Methods." Values for age represent mean \pm SEM.

* F:M ratio: $3: 2$; age: $3.8 \pm 1.5$ mo. (median age: $3.5 \mathrm{mo}$ ).

$\dagger$ F:M ratio: 2:3; age: $3.6 \pm 1.1$ mo. (median age: 3.5 mo).

samples classified as either clinically moderate or severe hypertrophy. The expression of MCD, ACC, and AMPK was assessed in two groups ( $n=5$ per group) in the 2-6 mo age range with either clinically moderate or severe hypertrophy (Table 2). No difference in the expression of MCD, ACC, or AMPK was observed between moderate and severe hypertrophy. However, there was a significant $(p=0.03)$ decrease in the ratio of P-AMPK (active) to total-AMPK in patients with severe hypertrophy versus patients with moderate hypertrophy (Table 3 ).

\section{DISCUSSION}

This study investigated the effects of age and myocardial hypertrophy on the expression of cardiac MCD, as well as expression and phosphorylation of cardiac ACC and AMPK. There was an age-dependent increase in the expression of MCD in ventricular biopsy samples obtained from pediatric patients ranging from 0.2 to 10 mo of age. In contrast, there was an age-dependent decrease in the expression of ACC and AMPK without any age-dependent effects on the phosphorylation of either enzyme in the newborn human heart. There was no effect of hypertrophy (severe hypertrophy versus moderate hypertrophy) with regards to the expression of $\mathrm{MCD}$, or the expression and phosphorylation of ACC. Although there was no difference

Table 3. Effects of myocardial hypertrophy on the expression of $M C D$, and the expression and phosphorylation of ACC and AMPK

\begin{tabular}{lccr}
\hline \multicolumn{1}{c}{ Enzyme } & $\begin{array}{c}\text { Moderate } \\
\text { hypertrophy }\end{array}$ & $\begin{array}{c}\text { Severe } \\
\text { hypertrophy }\end{array}$ & \multicolumn{1}{c}{$p$} \\
\hline MCD & $213.79 \pm 29.59$ & $194.71 \pm 14.40$ & $>0.99$ \\
ACC & $162.56 \pm 4.43$ & $161.33 \pm 3.64$ & 0.69 \\
P-ACC & $159.90 \pm 11.43$ & $154.63 \pm 4.45$ & 0.69 \\
P-ACC:ACC & $0.99 \pm 0.09$ & $0.96 \pm 0.03$ & $>0.99$ \\
AMPK & $119.55 \pm 5.64$ & $129.81 \pm 6.21$ & 0.31 \\
P-AMPK & $182.36 \pm 12.21$ & $150.86 \pm 9.47$ & 0.10 \\
P-AMPK:AMPK & $1.53 \pm 0.10$ & $1.17 \pm 0.08$ & $0.03^{*}$ \\
\hline
\end{tabular}

The expression of MCD, ACC and AMPK (arbitrary density units) was determined by densitometric analysis of western immunoblots of ventricular biopsy samples from patients with moderate hypertrophy $(n=5)$ and patients with severe hypertrophy $(n=5)$. Values represent means \pm SEM.

* Indicates significance at the $p<0.05$ level estimated using a MannWhitney $U$-test. in the expression of AMPK between severe and moderate hypertrophy, the phosphorylation of AMPK was reduced in the setting of severe hypertrophy. These data suggest that alterations in the expression of MCD, ACC, and AMPK likely underlie changes in malonyl CoA content (not assessed in this study), particularly as it relates to the maturation of fatty acid oxidation in the newborn human heart as it ages. Furthermore, these data suggest that alterations in AMPK phosphorylation (hence activation), but not AMPK expression may account for differences in the extent of maturation of fatty acid oxidation observed in differing degrees of myocardial hypertrophy in the newborn human heart.

Examination of MCD expression revealed an age dependent increase after birth. This increased expression likely regulates the maturation of fatty acid oxidation, as the increase in MCD content may increase the degradation of malonyl $\mathrm{CoA}$ and thus disinhibit CPT 1, the rate-limiting enzyme of mitochondrial fatty acid oxidation. This is supported by previous findings in the newborn rabbit heart, where there is a dramatic reduction in malonyl CoA content between 1 and $7 \mathrm{~d}$ of age that coincides with a 10-fold increase in fatty acid oxidation. MCD activity itself does not appear to change in the face of differences in fatty acid delivery to the newborn heart (27). Although we were unable to determine MCD activity in ventricular biopsies due to the limited nature of the samples, previous studies have demonstrated that MCD activity increases as the newborn heart increases in age (11). Although that study did not assess MCD expression (11), it does lend support the possible importance of changes in MCD expression underlying changes in MCD activity to thus regulate malonyl CoA content and the maturation of fatty acid oxidation in the newborn heart.

In contrast to MCD expression, there was an age-dependent decrease in the expression of ACC. Changes in ACC activity have been demonstrated to contribute to the maturation of fatty acid oxidation in hearts obtained from both newborn rabbits ( 1 and $7 \mathrm{~d}$ of age) $(9,17,28)$, as well as hearts obtained from newborn pigs (14 d of age) $(9,17,28)$. As we did not observe any age-dependent alteration in the phosphorylation of ACC (indicative of ACC inhibition), the data in this study suggests that in the newborn human heart the synthesis of malonyl CoA and maturation of fatty acid oxidation is not regulated by ACC activity, but is rather regulated by the extent of ACC expression. Examination of AMPK revealed an agedependent decrease in expression, without any age-dependent effect on phosphorylation (thus, activation) in the newborn human heart. Although this result contrasts with previous findings (19), in which AMPK expression increases during the course of maturation of fatty acid oxidation in hearts from newborn rabbits ( 1 and $7 \mathrm{~d}$ of age), it is consistent with the lack of an agedependent effect on ACC phosphorylation observed in this study. As AMPK contributes to the acute and rapid regulation of ACC activity via reversible phosphorylation and inhibition, these results further support that after birth the regulation of malonyl CoA content as it pertains to the maturation of fatty acid oxidation in newborn human heart is accomplished via expressional changes in both MCD and ACC.

The expression of MCD, ACC, and AMPK in a select group of patients of similar age revealed no differences between moderate and severe hypertrophy. Hypertrophy was categorized ac- 
cording to patient age and lesion type after ventricular biopsy, at which point right ventricular wall thickness was not influenced by in vivo cardiac hemodynamic and pressure parameters. Although a previous report describes a delay in the maturation of fatty acid oxidation in the hypertrophied newborn (up to $21 \mathrm{~d}$ of age) pig heart due in part to maintenance of ACC activity and decreased expression of the AMPK catalytic $\alpha$-subunit (17), the lack of available ventricular biopsy samples without any degree of hypertrophy precluded such an assessment in the present study. Interestingly, the expression and extent of AMPK phosphorylation did not differ between moderate and severe hypertrophy per se, however, there was a significant decease in PAMPK when normalized to the total amount of AMPK detected. This may suggest that the degree of hypertrophy can influence the phosphorylation and thus activation of AMPK. However, this effect of hypertrophy must be interpreted with caution as this difference, although statistically significant, may not be physiologically relevant as it arises due to nonsignificant changes in both total and phosphorylated AMPK between moderate and severe hypertrophy (Table 3).

Study limitations. Due to the nature of available ventricular biopsy samples (i.e. right ventricle), we were limited to assessing only the expression of MCD, ACC, and AMPK, as well as the phosphorylation of ACC and AMPK in the newborn human heart. Therefore, whether the expressional changes observed can be extrapolated to the left ventricle, and as such, whether these changes occur at the whole heart level remains unknown. Our previous report examining the maturation of fatty acid oxidation in the newborn pig heart ( $21 \mathrm{~d}$ of age) reveals that there is no difference in the content of malonyl $\mathrm{CoA}$, or the activities of either ACC or AMPK in the setting of volume overload hypertrophy between the right and left ventricles (17). However, there are currently no published reports characterizing possible differences in the regional expression of MCD, ACC, and AMPK between the right and left ventricles, as such this issue remains to be addressed. The limited quantity of available ventricular biopsy samples also restricted this study to assessing only the expression of MCD, ACC, and AMPK, as well as the phosphorylation of ACC and AMPK in the newborn human heart. As such, we were unable to directly characterize any age-dependent effects on the activity of each enzyme. With regards to AMPK expression and activity, there are two distinct catalytic $\alpha$-subunits $\left(\alpha_{1}\right.$ and $\left.\alpha_{2}\right)$. Previous studies have demonstrated that hypertrophy differentially affects the expression and activity of each catalytic subunit (28). As the AMPK- $\alpha$ antibody used in this study does not discriminate between $\alpha_{1}$ and $\alpha_{2}$ isoforms, it precludes insight regarding expressional changes between the catalytic isoforms in the presence of either moderate or severe hypertrophy. Furthermore, as all ventricular biopsy samples in this study displayed some degree of hypertrophy, there was no ideal "control" group for comparison.

In conclusion, this study has shown an age-dependent increase in the expression of MCD, as well as an age dependent decrease in the expression of ACC and AMPK in right ventricular biopsy samples obtained from infants undergoing surgery to correct various congenital heart defects. As there were no age-dependent changes in the phosphorylation of ACC and AMPK, it suggests the levels of expression, and not activity may be important regulators of the maturation of fatty acid oxidation in the newborn human heart in the presence of varying degrees of hypertrophy.

Acknowledgments. We thank Delores Poppe, R.N., R.D.C.S., R.D.M.S. for performing quantitative echocardiographic measurements.

\section{REFERENCES}

1. Dyck JR, Lopaschuk GD 2002 Malonyl CoA control of fatty acid oxidation in the ischemic heart. J Mol Cell Cardiol 34:1099-1109

2. Kantor PF, Lopaschuk GD, Opie LH 2001 Myocardial energy metabolism. In: Kurachi Y, Terzig A, Cohen MV, Sperelakis N (eds) Heart Physiology and Pathophysiology, 4E. New York: Academic Press, pp 543-569

3. Lopaschuk GD 2002 Metabolic abnormalities in the diabetic heart. Heart Fail Rev 7:149-159

4. Lopaschuk GD, Belke DD, Gamble J, Itoi T, Schonekess BO 1994 Regulation of fatty acid oxidation in the mammalian heart in health and disease. Biochim Biophys Acta 1213:263-276

5. Saddik M, Gamble J, Witters LA, Lopaschuk GD 1993 Acetyl-CoA carboxylase regulation of fatty acid oxidation in the heart. J Biol Chem 268:25836-25845

6. Girard J 1990 Metabolic adaptations to change of nutrition at birth. Biol Neonate 58:3-15

7. Fisher DJ, Heymann MA, Rudolph AM 1980 Myocardial oxygen and carbohydrate consumption in fetal lambs in utero and in adult sheep. Am J Physiol 238:H399-H405

8. Fisher DJ, Heymann MA, Rudolph AM 1981 Myocardial consumption of oxygen and carbohydrates in newborn sheep. Pediatr Res 15:843-846

9. Lopaschuk GD, Spafford MA 1990 Energy substrate utilization by isolated working hearts from newborn rabbits. Am J Physiol 258:H1274-H1280

10. Lopaschuk GD, Witters LA, Itoi T, Barr R, Barr A 1994 Acetyl-CoA carboxylase involvement in the rapid maturation of fatty acid oxidation in the newborn rabbit heart. J Biol Chem 269:25871-25878

11. Onay-Besikci A, Campbell FM, Hopkins TA, Dyck JR, Lopaschuk GD 2003 Relative importance of malonyl $\mathrm{CoA}$ and carnitine in maturation of fatty acid oxidation in newborn rabbit heart. Am J Physiol Heart Circ Physiol 284:H283-H289

12. McGarry JD, Leatherman GF, Foster DW 1978 Carnitine palmitoyltransferase I. The site of inhibition of hepatic fatty acid oxidation by malonyl-CoA. J Biol Chem 253:4128-4136

13. Reszko AE, Kasumov T, David F, Thomas KR, Jobbins KA, Cheng JF, Lopaschuk GD, Dyck JR, Diaz M, Des Rosiers C, Stanley WC, Brunengraber H 2004 Regulation of malonyl-CoA concentration and turnover in the normal heart. J Biol Chem 279:34298-34301

14. Longnus SL, Wambolt RB, Barr RL, Lopaschuk GD, Allard MF 2001 Regulation of myocardial fatty acid oxidation by substrate supply. Am J Physiol Heart Circ Physiol 281:H1561-H1567

15. Wang D, Buja LM, McMillin JB 1996 Acetyl coenzyme A carboxylase activity in neonatal rat cardiac myocytes in culture: citrate dependence and effects of hypoxia. Arch Biochem Biophys 325:249-255

16. Dyck JR, Barr AJ, Barr RL, Kolattukudy PE, Lopaschuk GD 1998 Characterization of cardiac malonyl-CoA decarboxylase and its putative role in regulating fatty acid oxidation. Am J Physiol 275:H2122-H2129

17. Kantor PF, Robertson MA, Coe JY, Lopaschuk GD 1999 Volume overload hypertrophy of the newborn heart slows the maturation of enzymes involved in the regulation of fatty acid metabolism. J Am Coll Cardiol 33:1724-1734

18. Makinde AO, Kantor PF, Lopaschuk GD 1998 Maturation of fatty acid and carbohydrate metabolism in the newborn heart. Mol Cell Biochem 188:49-56

19. Makinde AO, Gamble J, Lopaschuk GD 1997 Upregulation of 5'-AMP-activated protein kinase is responsible for the increase in myocardial fatty acid oxidation rates following birth in the newborn rabbit. Circ Res 80:482-489

20. Allard MF, Schonekess BO, Henning SL, English DR, Lopaschuk GD 1994 Contribution of oxidative metabolism and glycolysis to ATP production in hypertrophied hearts. Am J Physiol 267:H742-H750

21. el Alaoui-Talibi Z, Landormy S, Loireau A, Moravec J 1992 Fatty acid oxidation and mechanical performance of volume-overloaded rat hearts. Am J Physiol 262:H1068-H1074

22. Taegtmeyer H, Golfman L, Sharma S, Razeghi P, van Arsdall M 2004 Linking gene expression to function: metabolic flexibility in the normal and diseased heart. Ann $\mathrm{N}$ Y Acad Sci 1015:202-213

23. Lopaschuk GD 2001 Optimizing cardiac energy metabolism: how can fatty acid and carbohydrate metabolism be manipulated? Coron Artery Dis 12:S8-S11

24. Lopaschuk GD, Rebeyka IM, Allard MF 2002 Metabolic modulation: a means to mend a broken heart. Circulation 105:140-142

25. Sambandam N, Lopaschuk GD, Brownsey RW, Allard MF 2002 Energy metabolism in the hypertrophied heart. Heart Fail Rev 7:161-173

26. Stanley WC, Sabbah HN 2005 Metabolic therapy for ischemic heart disease: the rationale for inhibition of fatty acid oxidation. Heart Fail Rev 10:275-279

27. Onay-Besikci A, Sambandam N 2006 Malonyl CoA control of fatty acid oxidation in the newborn heart in response to increased fatty acid supply. Can J Physiol Pharmacol 84:1215-1222

28. Tian R, Musi N, D'Agostino J, Hirshman MF, Goodyear LJ 2001 Increased adenosine monophosphate-activated protein kinase activity in rat hearts with pressure-overload hypertrophy. Circulation 104:1664-1669 